\title{
An Analysis of the Correlation between Humeral Head Anterior Glide Posture and Elbow Joint Angle, Forward Head Posture and Glenohumeral Joint Range of Motion
}

\author{
Wontae Gong, PhD, $\mathrm{PT}^{1}$, Ilsub Jun, MSc, PT2², Yoorim Choi, MD, PT ${ }^{3)}$ \\ 1) Department of Physical Therapy, Korea Nazarene University \\ 2) Department of Rehabilitation Science, Graduate School, Daegu University: 201 Daegudae-lo, \\ Gyungsan-si, Kyoungbuk 712-714, Republic of Korea. TEL: +82 53-850-4653, FAX: +82 53-850-4359 \\ 3) Department of Physical Therapy, Korea Taegu Science University
}

\begin{abstract}
Purpose] The purpose of this study was to examine the correlation between the humeral head anterior glide posture (HHAGP) and elbow joint angle (EJA), forward head posture (FHP), and glenohumeral joint (GHJ) range of motion (ROM). [Subjects] The subjects were 47 college students (10 male, 37 female) in whom more than one third of the humeral head was anteriorly translated against the acromion. [Methods] Humeral head distance (HHD) was measured with vernier calipers and EJA and FHP were measured using NIH Image J software for lateral body imaging obtained with a digital camera. GHJ ROM was measured with a goniometer. [Results] Increases in HHD were accompanied by decreases in internal rotation (IR) and abduction (AB), while increases in forward head angle (FHA) accompanied decreases in EJA. Increases in EJA occurred together with increases in external rotation (ER). IR and ER increased with increases in AB. [Conclusion] HHD influences EJA, IR, and AB; therefore, HHD should be considered in therapeutic approaches for these factors.

Key words: Humeral head anterior glide posture, Elbow joint angle, Forward head posture
\end{abstract}

(This article was submitted Nov. 8, 2012, and was accepted Dec. 12, 2012)

\section{INTRODUCTION}

A proper static posture is an important element of health ${ }^{1)}$, and posture evaluation is essential for comparing and verifying the diagnosis and the efficacy of manipulative intervention ${ }^{2}$. An ideal shoulder joint alignment is less than one-third translation of the humeral head against the anterior acromion, with the antecubital crease placed anteriorly, the olecranon process located posteriorly, and the proximal and distal areas of the humerus placed in the same vertical plane ${ }^{3)}$. When the pectoralis major muscle (which pulls the humeral head anteriorly) acts superior to the subscapularis muscle (which pulls the humeral head posteriorly), an anterior protrusion of the humeral head occurs, which is called humeral head anterior glide posture (HHAGP) ${ }^{4}$.

In patients with HHAGP, more than one third of the humeral head protrudes from the anterior acromion and the humeral head is located more anteriorly than the distal humerus; i.e., the distal and proximal areas of the humeral head are not situated in the same vertical plane. Pain occurs in the anterior shoulder or anterior medial shoulder and increases during internal rotation, hyperextension, and horizontal internal rotation of the humerus. In the muscle recruitment pattern, the action by the pectoralis major

*To whom correspondence should be addressed.

E-mail: sub7475@hanmail.net muscle is predominant to that of the subscapularis muscle, and the humeral head is pulled anteriorly; therefore, the pectoralis major muscle and external rotation muscle are shortened and the internal rotation muscle is weakened and lengthened ${ }^{4}$. Without appropriate counterbalance by the subscapularis muscle, the pectoralis major muscle may cause the humeral head to slide forward excessively ${ }^{5,6}$. The most common resulting shift is an anterior translation of the humeral head, accompanied by medial rotation of the glenohumeral joint $(\mathrm{GHJ})^{3)}$.

Most studies of upper extremity alignment have investigated round shoulder posture (RSP) ${ }^{7,8)}$ forward head posture (FHP $)^{9)}$. No research has yet been conducted on the effects of muscular imbalance arising from by HHAGP on forward head angle, elbow joint angle, and GHJ range of motion (ROM). Accordingly, the aim of this study was to measure the humeral head distance (HHD), which is the standard for estimating HHAGP, and to examine the effects of HHD on the ranges of FHA, EJA, internal rotation (IR), external rotation (ER), and abduction (AB), in order to provide basic data for the assessment of patients' static postures and possible approaches for physical therapy.

\section{SUBJECTS AND METHODS}

The subjects for the present study were 47 students (10 males and 37 females) with HHAGP of D college in Daegu, Korea. Their average age, height, and weight were 
Table 1. Mean and standard deviation of measured values of HHD, FHA, IR, ER, and AB (M \pm SD)

\begin{tabular}{cccccc}
\hline HHD & FHA & EJA & IR & ER & AB \\
\hline $32.1 \pm 8.13$ & $38.5 \pm 6.6$ & $165.2 \pm 6.6$ & $41.7 \pm 10.4$ & $94.4 \pm 15.4$ & $83.5 \pm 13.3$ \\
\hline
\end{tabular}

HHD: humeral head distance; FHA: forward head angle; EJA: elbow joint angle; IR: internal rotation; ER: external rotation; AB: abduction

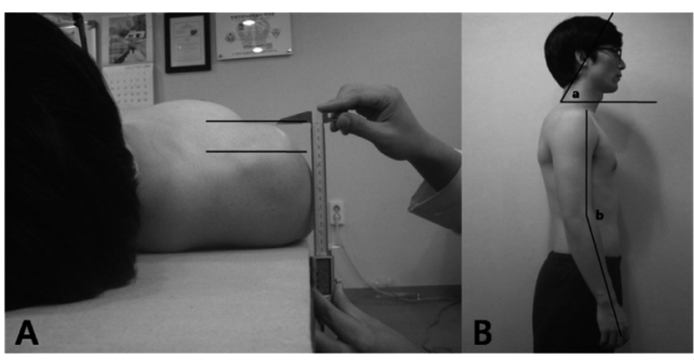

Fig. 1. Measurement of HHD, FHA, and EJA

A. Measurement of HHD taken with vernier caliper, B. Measurement of FHA(a) and EJA(b),

HHD: humeral head distance; FHA: forward head angle; EJA: elbow joint angle

$24.6 \pm 4.9$ years old, $163.8 \pm 6.4 \mathrm{~cm}$, and $54.1 \pm 8.3 \mathrm{~kg}$. The subjects had more than one third of the humeral head anteriorly translated against the acromion. Those who had received surgical treatment of the neck, shoulder, and elbow areas, who had a systemic disease, who had pain accompanied by fracture, sprain, or strain, or who were not able to maintain a standing position due to problems with the lower extremities, pelvis, or spine were excluded. The subjects sufficiently understood the purpose of this study and the overall content of the experiment, and they provided their written consent to their participation.

First, we measured the subjects' HHDs, and then their ROMs, FHAs, and EJAs were measured at the GHJ.

The HHD was measured with the subject lying in a relaxed position on a table, with both arms placed beside the trunk in the neutral position. The measurer then calculated the difference between the distance from the table and the peak of the humeral head, and the distance from the table and the acromion (Fig. 1A). The FHA and EJA were measured with the subject standing comfortably, while maintaining his or her vision horizontally, and relaxing the neck, shoulder, and upper extremity muscles. The lateral body view was photographed using an EOS650D (Canon, Japan) digital camera. Landmarks were placed on the tragus, the spinous process of the seventh cervical vertebrae, the acromion, the lateral epicondyle of the humerus, and the middle area between the radial styloid process and the ulnar head. The camera was installed in line with the subject's shoulder height and the distance between the camera and the measurer was $2 \mathrm{~m}$. The FHA between the vertical line passing through the spinous process of the seventh cervical vertebrae and the line passing through the spinous process of the seventh cervical vertebrae and the tragus was measured using NIH Image $\mathbf{J}$ software from the lateral body image
Table 2. Correlation of HHD, FHA, EA, IR, ER and AB

\begin{tabular}{lclllll}
\hline Category & HHD & FHA & EJA & IR & ER & AB \\
\hline HHD & 1 & & & & & \\
FHA & 0.29 & 1 & & & & \\
EJA & -0.48 & $-0.51^{*}$ & 1 & & & \\
IR & $-0.55^{*}$ & -0.23 & 0.47 & 1 & & \\
ER & -0.31 & -0.11 & $0.59^{*}$ & 0.42 & 1 & \\
AB & $-0.57^{*}$ & -0.11 & 0.22 & $0.49^{*}$ & $0.52^{*}$ & 1 \\
\hline$* \mathrm{p}<0.05$ & & & & & &
\end{tabular}

captured by the digital camera. A previous study asserts that a higher angle indicates a more severe forward head position ${ }^{9}$. EJA was determined by measuring the angle between the line connecting the acromion and the humerus lateral epicondyle and the line connecting the middle area of radial styloid process and the ulnar head and humerus lateral epicondyle (Fig. 1B).

The GHJ ROM was measured by two examiners. Examiner 1 measured ROM and examiner 2 prevented all compensatory actions. The measurement of shoulder abduction was performed with the subjects standing against a flat wall and maintaining extension of the elbow joint while restricting lateral flexion of the trunk. The subjects then conducted shoulder abduction and ROM was measured using a goniometer (USA) while the ulnar side of the forearm and the 5 th finger remained in contact with the wall ${ }^{10)}$. Internal and external rotation of the shoulder were measured with the subject in the supine position, with the GHJ at $90^{\circ}$ abduction and the elbow joint (EJ) at $90^{\circ}$ flexion, while the front shoulder joint was held to prevent compensatory action, and the rotational axis was fixed to prevent movement during rotation. The ROM was measured using a goniometer. All measurements were taken three times and averaged.

The data collected in this study were analyzed using SPSS 17.0, and the collected data are presented as the average and standard deviation. The correlations among HHD, FHA, EJA, IR, ER, and AB were investigated by calculating the Pearson correlation coefficient. The significance level was chosen as $\alpha=0.05$.

\section{RESULTS}

The mean \pm standard deviation values for HHD, FHA, EJA, IR, ER, and AB are presented in Table 1. Increases in HHD accompanied decreases in $\operatorname{IR}(\mathrm{r}=-0.55)$ and $\mathrm{AB}$ $(\mathrm{r}=-0.57)$, and increases in FHA accompanied decreases in EJA ( $r=-0.51)$. Decreases in EJA occurred together with decreases in $\mathrm{ER}(\mathrm{r}=0.59)$ and decreases in $\mathrm{AB}$ were associated with decreases in IR $(r=0.49)$ and in $E R(r=0.52)$ (Table 2). 


\section{DISCUSSION}

An abnormality in the complex structure of the cephalic, cervical, or humeral area or in the muscular balance surrounding the shoulder structure, or disruption of the maintenance of craniocervical posture, can give rise to pain, restriction of the ROM, and postural misalignment ${ }^{11)}$. Janda ${ }^{3)}$ observed that RSP and FHP increased in upper crossed syndrome patients. Shortening of the pectoralis minor muscle, which is attached to the coracoid process, anteriorly tilts the scapula, causing RSP, and FHP is characterized by increasing abnormality of the scapular alignment ${ }^{12,13)}$. A weak positive correlation was noted in the present study between HHD and FHA ( $r=0.29$ ), and HHD occurs due to the predominant action of the pectoralis major muscle ${ }^{4)}$. This muscle inserts at the humeral head; therefore, it has a greater engagement in the location of humerus than of the scapula, and ultimately, it does not greatly influence FHA.

The biceps brachii is involved in GHJ and EJ movement, but the active role of the long head of the biceps brachii muscle is not obvious, as it passively contributes to preventing upper and anterior displacement of the humeral head ${ }^{14,15)}$. In the present study, EJA decreased as HHD increased. Anterior translation of the humeral head is thought to deliver passive tension to the long head of the biceps brachii and to decrease the angle of the elbow joint. Hoang et al. ${ }^{16)}$ noted that the passive tension that occurs in response to changes in the location of one attachment point of multijoint muscles may affect other joints. In the present study, as HHD increased, IR and AB decreased in the GHJ. We consider this took place due to the change in the axis of the humeral head movement within the scapula glenoid cavity. Sahrmann et al. ${ }^{4)}$ reported that the posterior articular capsule was stiff in HHAGP, and that excessive anterior translational movement occurred during the abduction of the shoulder joint. Kibler et al. ${ }^{17)}$ asserted that stiffness of the posterior articular capsule resulted in a greater reduction in the ROM of IR than of ER. Therefore, a therapeutic approach to GHJ aimed at increasing the ROM of IR and AB, or at improving EJA, should take into account the location of the humeral head.

\section{REFERENCES}

1) McEvoy MP, Grimmer K: Reliability of upright posture measurements in primary school children. BMC Musculoskelet Disord, 2005, 6: 35. [Medline]

2) Ferreira EA, Duarte M, Maldonado EP, et al.: Quantitative assessment of postural alignment in young adults based on photographs of anterior, posterior, and lateral views. J Manipulative Physiol Ther, 2011, 34: 371-380. [Medline] [CrossRef]

3) Page P, Frank C: Lardner. Assessment and treatment of muscle imbalance; The Janda Approach. Champaign: Human Kinetics, 2010, pp 69-70.

4) Sahrmann SA: Diagnosis and treatment of movement impairment syndrome. St Louis: Mosby, 2002, pp 231-234.

5) Cordasco FA, Wolfe IN, Wootten ME, et al.: An electromyographic analysis of the shoulder during a medicine ball rehabilitation program. Am J Sports Med, 1996, 24: 386-392. [Medline] [CrossRef]

6) Neumann DA: Kinesiology of the musculoskeletal system; foundations for physical rehabilitation. St Louis: Mosby, 2002, pp 129-130.

7) Borstad JD: Measurement of pectoralis miner muscle length; validation and clinical application. J Orthop Sports Phys Ther, 2008, 38: 169-174. [Medline]

8) Borstad JD, Ludewig PM: The effect of long versus short pertoralis miner resting length on scapular kinematics in healthy individuals. J Orthop Sports Phys Ther, 2005, 35: 227-238. [Medline]

9) Thigpen CA, Padua DA, Michener LA, et al.: Head and shoulder posture affect scapular mechanics and muscle activity in overhead tasks. J Electromyogr Kinesiol, 2010, 20: 701-709. [Medline] [CrossRef]

10) Gong WT, Lee HM, Lee YM: Effects of Gong's mobilization applied to shoulder joint on shoulder abduction. J Phys Ther, 2011, 23: 391-393. [CrossRef]

11) Bae SS, Kim BJ, Lee KH: A study of muscle imbalance of head, cervical and shoulder region. J Korean Soc Phys Ther, 2001, 13: 769-776.

12) Lukasiewicz AC, McClure P, Michener L, et al.: Comparison of 3-dimensional scapular position and orientation between subjiects with and without shoulder impingement. J Orthop Sports Phys Ther, 1999, 29: 574-583. [Medline]

13) Borstad JD, Ludewig PM: Comparison of three stretches for the pectoralis minor muscle. J Shoulder Elbow Surg, 2006, 15: 324-330. [Medline] [CrossRef]

14) Krupp RJ, Kevern MA, Gaines MD, et al.: Long head of the biceps tendon pain: differential diagnosis and treatment. J Orthop Sports Phys Ther, 2009, 39: 55-70. [Medline]

15) Rodosky MW, Harner CD, Fu FH: The role of the long head of the biceps muscle and superior glenoid labrum in anterior stability of the shoulder. Am J Sports Med, 1994, 22: 121-130. [Medline] [CrossRef]

16) Hoang PD, Herbert RD, Gorman RB, et al.: A new method for measuring passive length-tension properties of human gastrocnemius muscle in vivo. J Biomech, 2005, 38: 1333-1341. [Medline] [CrossRef]

17) Kibler WB, Sciascia A, Thomas SJ: Glenohumeral Internal Rotation Deficit: Pathogenesis and Response to Acute Throwing. Sports Med Arthrosc, 2012, 20: 34-38. [Medline] [CrossRef] 